\title{
A NEW PROOF OF SEMICONTINUITY BY YOUNG MEASURES AND AN APPROXIMATION THEOREM IN ORLICZ-SOBOLEV SPACES
}

\author{
BARBARA BIANCONI
}

Received 6 December 2002

We give a new approach to study the lower semicontinuity properties of nonautonomous variational integrals whose energy densities satisfy general growth conditions. We apply the theory of Young measures and properties of OrliczSobolev spaces to prove semicontinuity result.

\section{Introduction}

In the last years there has been a particular interest in the research of minimizers of nonautonomous variational integrals whose energy densities satisfy general growth conditions such as

$$
0 \leq f(x, s, z) \leq E(x, s)\{1+\Phi(|z|)\}
$$

where $f=f(x, s, z)$ is a real Carathéodory function defined in $\Omega \times \mathbb{R}^{m} \times \mathbb{R}^{m n}$, quasiconvex with respect to $z$, in Morrey's sense, that is, for every $\left(x_{0}, s_{0}, z_{0}\right) \in$ $\Omega \times \mathbb{R}^{m} \times \mathbb{R}^{m n}$ and $\varphi \in C_{0}^{\infty}\left(\Omega, \mathbb{R}^{m}\right)$ there holds

$$
f\left(x_{0}, s_{0}, z_{0}\right)|\Omega| \leq \int_{\Omega} f\left(x_{0}, s_{0}, z_{0}+D \varphi(y)\right) d y .
$$

The function $E: \Omega \times \mathbb{R}^{m} \rightarrow \mathbb{R}$ is a positive Carathéodory's and $\Phi$ is an $N$-function.

A convex function $\Phi:[0,+\infty[\rightarrow[0,+\infty[$ is called $N$-function if it satisfies the following conditions: $\Phi(0)=0, \Phi(t)>0$ for $t>0$, and

$$
\lim _{t \rightarrow 0} \frac{\Phi(t)}{t}=0, \quad \lim _{t \rightarrow+\infty} \frac{\Phi(t)}{t}=+\infty .
$$

When $\Phi(z)=z^{p}$, we say that $f$ verifies standard growth conditions.

Copyright (C) 2003 Hindawi Publishing Corporation Abstract and Applied Analysis 2003:15 (2003) 881-898 2000 Mathematics Subject Classification: 49J45

URL: http://dx.doi.org/10.1155/S1085337503303045 
The study of nonautonomous variational integrals is relevant for studying the applications in the theory of elastic and magnetostatic material behaviors. Often a starting point is the necessary and sufficient conditions ensuring sequential weak lower semicontinuity of the functional

$$
F(u)=\int_{\Omega} f(x, u(x), D u(x)) d x .
$$

Acerbi and Fusco [5] and Marcellini [11] give a well-known weak lower semicontinuity theorem, when $f$ is quasiconvex in Morrey's sense and satisfies the standard growth.

Theorem 1.1. Let $\Omega$ be an open set in $\mathbb{R}^{m}$. Assume that $f=f(x, s, z)$ is a real Carathéodory function defined in $\Omega \times \mathbb{R}^{m} \times \mathbb{R}^{m n}$, quasiconvex with respect to $z$ in Morrey's sense, and such that

$$
0 \leq f(x, s, z) \leq a(x)+c\left(|s|^{p}+|z|^{p}\right) \quad \text { for a.e. } x \in \Omega, \forall s \in \mathbb{R}^{m}, \forall z \in \mathbb{R}^{m n},
$$

where $c$ is a positive constant, $p \geq 1$, and $a \in L_{\mathrm{loc}}^{1}(\Omega)$.

Then the functional

$$
u \in W^{1, p}\left(\Omega ; \mathbb{R}^{m}\right) \longrightarrow \int_{\Omega} f(x, u(x), D u(x)) d x
$$

is sequentially lower semicontinuous in the weak topology of $W^{1, p}\left(\Omega ; \mathbb{R}^{m}\right)$.

In [4], the result has been generalized by Bianconi et al. for general growth (1.1) and the lower semicontinuity in the weak* topology of the Orlicz-Sobolev spaces is proved.

In some physical problems, there may be situations where we need to identify $\lim _{n \rightarrow \infty} F\left(u_{n}\right)$ for an oscillatory sequence $\left\{u_{n}\right\}$ which does not minimize the energy. Consequently, this will entail a full characterization of the Young measure generated by the sequence under consideration.

In [7], there is a new proof of Theorem 1.1 by using Young measures. In this setting the semicontinuity is a direct consequence of the Jensen inequality.

In this paper, we give a new proof of the lower semicontinuity for quasiconvex integrals satisfying (1.1) in the framework of Young measures.

The first step is the Jensen-type inequality for Young measures in OrliczSobolev spaces.

Theorem 1.2. Let $\Omega \subset \mathbb{R}^{n}$ be a bounded set, $u^{j} \in W^{1, \Phi, 1}\left(\Omega, \mathbb{R}^{m}\right)$, and $\Phi \in \Delta_{2} \cap$ $\nabla_{2}$. Suppose that $u^{j} \rightarrow u$ in $L_{\text {loc }}^{1}$ norm and $\liminf _{j} \int_{\Omega} \Phi\left(\left|D u^{j}(x)\right|\right) d x<+\infty$.

Let $f: \Omega \times \mathbb{R}^{m n} \rightarrow[0,+\infty]$ verifying that

(a) $f(x, \lambda)$ is a Carathéodory function,

(b) a measurable function $E: \Omega \rightarrow \mathbb{R}$ exists such that for almost every $x \in \Omega$ and for all $\lambda \in \mathbb{R}^{m n}, f(x, \lambda) \leq E(x)(1+\Phi(|\lambda|))$ holds, 
(c) the function $\lambda \rightarrow f(x, \lambda)$ is quasiconvex for almost every $x \in \Omega$.

Then

$$
\begin{aligned}
f\left(x, \int_{\mathbb{R}^{m n}} \lambda d \nu_{x}(\lambda)\right) & \leq \int_{\mathbb{R}^{m n}} f(x, \lambda) d \nu_{x}(\lambda), \\
D u(x) & =\int_{\mathbb{R}^{m n}} \lambda d \nu_{x}(\lambda),
\end{aligned}
$$

where $\left\{\nu_{x}\right\}_{x \in \Omega}$ is the Young measure generated by a subsequence of $\left\{D u^{j}\right\}_{j \in \mathbb{N}}$.

In the proof of the Jensen's inequality for Young measures, an approximation theorem is fundamental which is an improvement of the result obtained by Acerbi and Fusco in [1] in the framework of Orlicz-Sobolev spaces.

Theorem 1.3. Let $\Omega \subseteq \mathbb{R}^{n}$ be the unit ball and $u \in W^{1, \Phi, 1}\left(\Omega, \mathbb{R}^{m}\right)$ with $\Phi$ a generic $N$-function, then for every constant $h>0$, there exist a function $u_{h} \in \operatorname{Lip}(\Omega$, $\left.\mathbb{R}^{m}\right)$ and a closed set $F_{h} \subset \Omega$ such that

(i) $\left\|\Phi\left(\nabla u_{h}\right)\right\|_{L^{\infty}\left(\Omega, \mathbb{R}^{m}\right)} \leq \Phi(h)$,

(ii) $\nabla u=\nabla u_{h}$ a.e. in $F_{h}$,

(iii) $\lim _{h \rightarrow+\infty}\left|\Omega \backslash F_{h}\right|=0$,

(iv) if $\Phi \in \Delta_{2} \cap \nabla_{2}$, then $\lim _{h \rightarrow+\infty} \Phi(h)\left|\Omega \backslash F_{h}\right|=0$.

The Jensen inequality is the main tool of the following theorem.

Theorem 1.4. Let $\Omega \subseteq \mathbb{R}^{n}$ be a bounded set and let $f: \Omega \times \mathbb{R}^{m} \times \mathbb{R}^{m n} \rightarrow[0,+\infty]$ with the following properties:

(1) $f(x, s, \lambda)$ is a Carathéodory function,

(2) a Carathéodory function $E(\cdot, \cdot)$ exists such that, for a.e. $x$ and for almost $(s, \lambda), f(x, s, \lambda) \leq E(x, s)(1+\Phi(|\lambda|))$, where $\Phi$ is an $N$-function with $\Phi \in$ $\Delta_{2} \cap \nabla_{2}$,

(3) for a.e. $x$ and for all $s$, the mapping $\lambda \mapsto f(x, s, \lambda)$ is quasiconvex.

Then for every $\left\{u^{j}\right\}_{j \in \mathbb{N}}, u^{j} \in W^{1, \Phi, 1}\left(\Omega, \mathbb{R}^{m}\right)$ such that $u^{j} \rightarrow u$ in $L_{\mathrm{loc}}^{1}\left(\Omega, \mathbb{R}^{m}\right)$ and $\liminf _{j} \int_{\Omega} \Phi\left(\left|D u^{j}(x)\right|\right) d x<+\infty$,

$$
I(u) \leq \liminf _{j} I\left(u^{j}\right)
$$

Proof. Let $\alpha=\liminf _{j} I\left(u^{j}\right)$. If $\alpha=+\infty$, the assertion is satisfied. Suppose that $\alpha<+\infty$. In this case the sequence $\left\{f\left(x, u^{j}(x), D u^{j}(x)\right)\right\}_{j \in \mathbb{N}}$ is bounded in $L^{1}(\Omega)$. Then we can find a subsequence $\left\{u^{l}\right\}_{l}$ of $\left\{u^{j}\right\}_{j \in \mathbb{N}}$ with the following properties:

(1) $I\left(u^{l}\right) \rightarrow \alpha$ as $l \rightarrow+\infty$,

(2) $\left\{D u^{l}\right\}_{l}$ generates the Young measure $\left\{v_{x}\right\}_{x \in \Omega}$,

(3) there exists a family $\left\{E_{k}\right\}_{k \in \mathbb{N}}$ of sets such that $\left|E_{k}\right| \rightarrow 0$ as $k \rightarrow+\infty$ and $\left\{f\left(x, u^{l}(x), D u^{l}(x)\right)\right\}_{l}$ is weakly convergent in $L^{1}\left(\Omega \backslash E_{k}\right)$ to $\int_{\mathbb{R}^{m n}} f(x$, $u(x), \lambda) d v_{x}(\lambda)$. 
Since $f_{u}(x, \lambda)=f(x, u(x), \lambda)$ satisfies the assumptions of Theorem 1.2, we have

$$
\int_{\mathbb{R}^{m n}} f_{u}(x, \lambda) d v_{x}(\lambda) \geq f_{u}(x, D u(x))
$$

hence

$$
\int_{\mathbb{R}^{m n}} f(x, u(x), \lambda) d v_{x}(\lambda) \geq f(x, u(x), D u(x))
$$

for a.e. $x$. Now it suffices to note that

$$
\begin{aligned}
\alpha & =\liminf _{j \rightarrow+\infty} I\left(u^{j}\right)=\lim _{l \rightarrow+\infty} \int_{\Omega} f\left(x, u^{l}(x), D u^{l}(x)\right) d x \\
& \geq \lim _{l \rightarrow+\infty} \int_{\Omega \backslash E_{k}} f\left(x, u^{l}(x), D u^{l}(x)\right) d x .
\end{aligned}
$$

The sequence $f\left(x, u^{l}(x), D u^{l}(x)\right)$ is weakly convergent in $L^{1}\left(\Omega \backslash E_{k}\right)$, then by dominate convergence

$$
\begin{aligned}
\lim _{l \rightarrow+\infty} \int_{\Omega \backslash E_{k}} f\left(x, u^{l}(x), D u^{l}(x)\right) d x & =\int_{\Omega \backslash E_{k}} \int_{\mathbb{R}^{m n}} f(x, u(x), \lambda) d v_{x}(\lambda) d x \\
& \geq \int_{\Omega \backslash E_{k}} f(x, u(x), D u(x)) d x \\
& =I(u)_{\left.\right|_{\Omega \backslash E_{k}}},
\end{aligned}
$$

the last inequality holds for Theorem 1.2 . Now by the fact that $\left|E_{k}\right| \rightarrow 0$, we have that in $\Omega,(1.9)$ is true, then

$$
\liminf _{j \rightarrow+\infty} I\left(u^{j}\right) \geq I(u)
$$

\section{Notations and preliminaries}

We denote by $\langle\cdot, \cdot\rangle$ the Euclidean scalar product in $\mathbb{R}^{n}$ and by $|\cdot|$ the usual Euclidean norm. Throughout the paper, $\Omega$ denotes an open and bounded subset of $\mathbb{R}^{n}$ with Lipschitz boundary. We denote by $\mid$ the Lebesgue measure on $\mathbb{R}^{n}$ and the notation a.e. stands for almost everywhere with respect to Lebesgue measure. We use standard notations for spaces of classically differentiable functions, Lebesgue and Sobolev spaces.

We recall some definitions and known properties of $\mathrm{N}$-functions and Orlicz spaces (see $[9,14]$ ).

In the sequel, we will often use the following convexity inequality: for every $s$, $t \in \mathbb{R}$ and $\lambda>1$,

$$
\Phi(s+t) \leq \frac{1}{\lambda} \Phi(\lambda s)+\left(1-\frac{1}{\lambda}\right) \Phi\left(\frac{\lambda}{\lambda-1} t\right)
$$

Now we will consider a special class of $N$-functions. 
Definition 2.1. An $N$-function $\Phi$ satisfies the $\Delta_{2}$ condition, that is $\Phi \in \Delta_{2}$, if there exist $r>1$ and $t_{0} \geq 0$ such that for every $t \geq t_{0}$ and $\lambda>1$ there holds

$$
\Phi(\lambda t) \leq \lambda^{r} \Phi(t)
$$

Definition 2.2. An $N$-function $\Phi$ satisfies the $\nabla_{2}$ condition, that is $\Phi \in \nabla_{2}$, if there exist $r>1$ and $t_{1} \geq 0$ such that for every $t \geq t_{1}$ and $k>1$ there holds

$$
\Phi(k t) \geq k^{r} \Phi(t)
$$

For further properties of $N$-functions of classes $\Delta_{2}$ and $\nabla_{2}$, see $[2,9,10,14]$. Let $\Omega$ be an open bounded set of $\mathbb{R}^{n}$, the $\operatorname{Orlicz}$ class $K^{\Phi}\left(\Omega, \mathbb{R}^{m}\right)$ is the set of all equivalence classes modulo equality a.e. in $\Omega$ of measurable functions $u: \Omega \rightarrow$ $\mathbb{R}^{m}$ satisfying

$$
\int_{\Omega} \Phi(|u|) d x<+\infty
$$

The Orlicz space $L^{\Phi}\left(\Omega, \mathbb{R}^{m}\right)$ is defined to be the linear hull of $K^{\Phi}\left(\Omega, \mathbb{R}^{m}\right)$.

The Orlicz-Sobolev space $W^{1} L^{\Phi}\left(\Omega, \mathbb{R}^{m}\right)$ is defined to be the set of all functions in $L^{\Phi}\left(\Omega, \mathbb{R}^{m}\right)$ whose first-order distributional derivatives are in $L^{\Phi}\left(\Omega, \mathbb{R}^{m}\right)$. In the sequel, for a fixed $\lambda>0$ we will consider the convex functional set

$$
W^{1, \Phi, \lambda}\left(\Omega, \mathbb{R}^{m}\right)=\left\{u \in W^{1,1}\left(\Omega, \mathbb{R}^{m}\right): \int_{\Omega} \Phi(\lambda|D u|) d x<+\infty\right\} .
$$

\section{An approximation theorem}

In this section, we give an approximation theorem for functions in $W^{1, \Phi, 1}$; we will use the properties of the maximal function, some of which are related with the properties of $N$-functions. For details see [15].

Definition 3.1. For every $f \in L_{\mathrm{loc}}^{1}\left(\mathbb{R}^{n}\right)$, set

$$
M f(x)=\sup _{r>0} \frac{1}{|B(x, r)|} \int_{B(x, r)}|f(y)| d y,
$$

and if $f \in W_{\text {loc }}^{1,1}\left(\mathbb{R}^{n}\right)$, set $M^{\prime} f(x)=M(|\nabla f|)$, that is,

$$
M^{\prime} f(x)=\sum_{i=1}^{n} M f_{x_{i}}(x) .
$$

We state particular properties for the maximal function (see $[8,12])$.

TheOrem 3.2. Let $\Phi \in \Delta_{2}$ be an $N$-function and $f$ a given positive function in $L_{\text {loc }}^{1}\left(\mathbb{R}^{n}\right)$.

Then if there exists a constant $a>1$ such that

$$
\Phi(t)<\frac{1}{2 a} \Phi(a t), \quad t \geq 0,
$$


886 Semicontinuity by Young measures

then

$$
\int_{\mathbb{R}^{n}} \Phi(M f(x)) d x \leq c \int_{\mathbb{R}^{n}} \Phi(f(x)) d x
$$

The maximal function $M^{\prime}$ permits to control the difference quotient of $u \in$ $W_{\text {loc }}^{1,1}\left(\mathbb{R}^{n}\right)$ outside a set of small measure.

Definition 3.3. Set, for any $u \in W_{\text {loc }}^{1,1}\left(\mathbb{R}^{n}\right)$ and for any $\lambda>0$,

$$
H_{\lambda, u}=\left\{x \in \mathbb{R}^{n}: M^{\prime} u(x)<\lambda\right\}
$$

Lemma 3.4. There exists a constant $c_{1}=c_{1}(n)$ such that, for every $u \in C_{0}^{\infty}\left(\mathbb{R}^{n}, \mathbb{R}^{m}\right)$,

$$
\frac{|u(x)-u(y)|}{|x-y|} \leq c_{1} \lambda \quad \forall x, y \in H_{\lambda, u}
$$

We now give other properties which relate the maximal function and the $\mathrm{N}$ function.

Lemma 3.5. Let $\Phi$ be an $N$-function, then for every $f \in L^{1}\left(\mathbb{R}^{n}\right)$ and for every constant $\lambda>0$,

$$
\Phi(\lambda)\left|\left\{x \in \mathbb{R}^{n}: M f(x) \geq \lambda\right\}\right| \leq \int_{\mathbb{R}^{n}} \Phi(f(x)) d x
$$

Proof. By the Jensen inequality applied to the convex function $\Phi$, we obtain

$$
\Phi(M f(x)) \leq M(\Phi(f(x)))
$$

for the monotonicity of $\Phi$ there is

$$
\begin{aligned}
\Phi(\lambda)\left|\left\{x \in \mathbb{R}^{n}: M f(x) \geq \lambda\right\}\right| & =\Phi(\lambda)\left|\left\{x \in \mathbb{R}^{n}: \Phi(M f(x)) \geq \phi(\lambda)\right\}\right| \\
& \leq \Phi(\lambda)\left|\left\{x \in \mathbb{R}^{n}: M(\Phi(f(x))) \geq \Phi(\lambda)\right\}\right| \\
& \leq \int_{\mathbb{R}^{n}} \Phi(f(x)) d x
\end{aligned}
$$

The last inequality is a property of the maximal operator $M$ [15].

In the sequel, we will use the following approximation result (for the proof see $[6])$. 
LEMMA 3.6. Let $\Omega$ be a regular open set of $\mathbb{R}^{n}$; if $u \in W^{1, \Phi, 1}\left(\Omega, \mathbb{R}^{m}\right)$, there exist a constant $\sigma>0$ and a sequence $u_{k} \in C_{0}^{\infty}\left(\Omega, \mathbb{R}^{m}\right)$ which converges modularly to $u$ : $u_{k} \underset{\bmod }{\stackrel{\sigma}{\longrightarrow}} u$, that is,

$$
\lim _{k \rightarrow+\infty} \int_{\Omega} \Phi\left(\frac{\left|D u(x)-D u_{k}(x)\right|}{\sigma}\right) d x=0
$$

Remark 3.7. In [6] the last result was proved only in the scalar case, but it is easy to show that it holds in general.

Now we have all the necessary ingredients to prove the approximation theorem.

Proof of Thereom 1.3. Let $u \in W^{1, \Phi, 1}\left(\Omega, \mathbb{R}^{m}\right), u_{k} \in C_{0}^{\infty}\left(\Omega, \mathbb{R}^{m}\right)$, and let $\sigma$ be as in Lemma 3.6.

In this framework we prove that if $f_{k} \in C_{0}^{\infty}\left(\mathbb{R}^{n}, \mathbb{R}^{m}\right)$ and $f \in W^{1, \Phi, 1}\left(\mathbb{R}^{n}, \mathbb{R}^{m}\right)$ with $f_{k} \underset{\bmod }{\stackrel{\sigma}{\longrightarrow}} f$, then a subsequence, which we will denote by $f_{k}$, exists, such that $M^{\prime} f_{k} \rightarrow M^{\prime} f$ in measure.

In fact, by the property $\left|M g_{1}(x)-M g_{2}(x)\right| \leq M\left(g_{1}-g_{2}\right)(x)$, for $\alpha>0$, there is

$$
\begin{aligned}
\mid\{x & \left.\in \mathbb{R}^{n}:\left|M^{\prime} f(x)-M^{\prime} f_{k}(x)\right| \geq \sigma \alpha\right\} \mid \\
& \leq\left|\left\{x \in \mathbb{R}^{n}: M\left(\frac{\left|D f(x)-D f_{k}(x)\right|}{\sigma}\right) \geq \alpha\right\}\right| ;
\end{aligned}
$$

applying Lemma 3.5 in (3.11) and the modular convergence, we have

$$
\begin{aligned}
\mid\{x & \left.\in \mathbb{R}^{n}:\left|M^{\prime} f(x)-M^{\prime} f_{k}(x)\right| \geq \sigma \alpha\right\} \mid \\
& \leq \frac{1}{\Phi(\alpha)} \int_{\mathbb{R}^{n}} \Phi\left(\frac{\left|D f(x)-D f_{k}(x)\right|}{\sigma}\right) d x \stackrel{k \rightarrow+\infty}{\longrightarrow} 0
\end{aligned}
$$

therefore, $M^{\prime} f_{k} \rightarrow M^{\prime} f$ in measure, then a subsequence $M^{\prime} f_{k_{j}}$ of $M^{\prime} f_{k}$, which we will denote by $M^{\prime} f_{k}$, exists such that $M^{\prime} f_{k} \rightarrow M^{\prime} f$ a.e. in $\mathbb{R}^{n}$.

Let $E \subset \mathbb{R}^{n}$ be the set with $|E|=0$ and

$$
\mathbb{R}^{n} \backslash E=\left\{x \in \mathbb{R}^{n}: f_{k}(x) \longrightarrow f(x), M^{\prime} f_{k}(x) \longrightarrow M^{\prime} f(x)\right\}
$$

We define another sequence as

$$
v_{k}(x):=\frac{1}{1+\sigma} u_{k}(x) \quad \forall k \in \mathbb{N} .
$$


Then $v_{k} \in C_{0}^{\infty}\left(\Omega, \mathbb{R}^{m}\right)$ for all $k>0$. Let $v_{k}^{*}$ be the natural extension of $v_{k}$ on $\mathbb{R}^{n}$, still denoted with $v_{k}$, then compute

$$
\begin{aligned}
\int_{\mathbb{R}^{n}} \Phi\left(\left|D v_{k}(x)\right|\right) d x \leq & \int_{\Omega} \frac{\sigma}{1+\sigma} \Phi\left(\left|D u_{k}(x)-D u(x)\right|\right) d x \\
& +\int_{\Omega} \frac{1}{1+\sigma} \Phi(|D u(x)|) d x \\
= & \frac{\sigma}{1+\sigma} \int_{\Omega} \Phi\left(\frac{\left|D u_{k}(x)-D u(x)\right|}{\sigma}\right) d x \\
& +\frac{1}{1+\sigma} \int_{\Omega} \Phi(|D u(x)|) d x
\end{aligned}
$$

By the modular convergence and since $u \in W^{1, \Phi, 1}\left(\Omega, \mathbb{R}^{m}\right)$, we have

$$
\int_{\mathbb{R}^{n}} \Phi\left(\left|D v_{k}(x)\right|\right) d x<\Gamma<+\infty
$$

therefore, $v_{k} \in W^{1, \Phi, 1}\left(\mathbb{R}^{n}, \mathbb{R}^{m}\right)$.

For $h>0$, define

$$
\Omega_{h, u}=\left\{x \in \Omega: M^{\prime} u(x)<\frac{h}{c_{1}}\right\}
$$

where $c_{1}$ is the constant of Lemma 3.4.

Since $M^{\prime} u_{k} \rightarrow M^{\prime} u$ a.e. on $\Omega$, then a constant $k_{0}$ exists such that, for every $k>k_{0}$,

$$
M^{\prime} u_{k}(x)<\frac{h}{c_{1}} \quad \text { a.e. } x \in \Omega_{h, u}
$$

and for Lemma 3.4

$$
\frac{\left|u_{k}(x)-u_{k}(y)\right|}{|x-y|} \leq c_{1} \frac{h}{c_{1}}=h \quad \text { a.e. } x, y \in \Omega_{h, u} .
$$

As $k \rightarrow+\infty$ we obtain

$$
\frac{|u(x)-u(y)|}{|x-y|} \leq h \quad \text { a.e. } x, y \in \Omega_{h, u},
$$

and we can conclude that the function $u$ is $h$-Lipschitz continuous in $\Omega_{h, u}$.

Furthermore, note that

$$
\begin{aligned}
\Omega \backslash \Omega_{h, u} & =\left\{x \in \Omega: M^{\prime} u(x) \geq \frac{h}{c_{1}}\right\} \\
& =\left\{x \in \Omega: M^{\prime} u(x)-M^{\prime} u_{k}(x)+M^{\prime} u_{k}(x) \geq \frac{h}{c_{1}}\right\}
\end{aligned}
$$




$$
\begin{aligned}
& \subset\left\{x \in \Omega:\left|M^{\prime} u(x)-M^{\prime} u_{k}(x)\right|+M^{\prime} u_{k}(x) \geq \frac{h}{c_{1}}\right\} \\
& \subset\left\{x \in \Omega:\left|M^{\prime} u(x)-M^{\prime} u_{k}(x)\right| \geq \frac{h}{2 c_{1}}\right\} \\
& \cup\left\{x \in \Omega: M^{\prime} u_{k}(x) \geq \frac{h}{2 c_{1}}\right\},
\end{aligned}
$$

and then

$$
\begin{aligned}
\left|\Omega \backslash \Omega_{h, u}\right| \leq & \left|\left\{x \in \Omega:\left|M^{\prime} u(x)-M^{\prime} u_{k}(x)\right| \geq \frac{h}{2 c_{1}}\right\}\right| \\
& +\left|\left\{x \in \Omega: M^{\prime} u_{k}(x) \geq \frac{h}{2 c_{1}}\right\}\right| .
\end{aligned}
$$

The first term becomes

$$
\begin{aligned}
\mid\{x & \left.\in \Omega:\left|M^{\prime} u(x)-M^{\prime} u_{k}(x)\right| \geq \frac{h}{2 c_{1}}\right\} \mid \\
& =\left|\left\{x \in \Omega: \frac{\left|M^{\prime} u(x)-M^{\prime} u_{k}(x)\right|}{\sigma} \geq \frac{h}{2 c_{1} \sigma}\right\}\right| \\
& \leq \frac{1}{\Phi\left(h / 2 c_{1} \sigma\right)} \int_{\Omega} \Phi\left(\frac{\left|D u_{k}(x)-D u(x)\right|}{\sigma}\right) d x
\end{aligned}
$$

for the second term, we compute

$$
\begin{aligned}
\left|\left\{x \in \Omega: M^{\prime} u_{k}(x) \geq \frac{h}{2 c_{1}}\right\}\right| & =\left|\left\{x \in \Omega: M^{\prime} v_{k}(x) \geq \frac{h}{2 c_{1}(1+\sigma)}\right\}\right| \\
& \leq\left|\left\{x \in \mathbb{R}^{n}: M\left(\left|D v_{k}(x)\right|\right) \geq \frac{h}{2 c_{1}(1+\sigma)}\right\}\right| \\
& \leq \frac{1}{\Phi\left(h / 2 c_{1}(1+\sigma)\right)} \int_{\mathbb{R}^{n}} \Phi\left(\left|D v_{k}(x)\right|\right) d x \\
& \leq \frac{\Gamma}{\Phi\left(h / 2 c_{1}(1+\sigma)\right)} .
\end{aligned}
$$

By (3.23) and (3.24), we have

$$
\begin{aligned}
\left|\Omega \backslash \Omega_{h, u}\right| \leq & \frac{1}{\Phi\left(h /\left(2 c_{1} \sigma\right)\right)} \int_{\mathbb{R}^{n}} \Phi\left(\frac{\left|D u_{k}(x)-D u(x)\right|}{\sigma}\right) d x \\
& +\frac{\Gamma}{\Phi\left(h / 2 c_{1}(1+\sigma)\right)} .
\end{aligned}
$$


As is well known, there exists an $h$-Lipschitzian function $u_{h}: \Omega \rightarrow \mathbb{R}^{m}$ with $u_{h} \equiv$ $u$ a.e. in $\Omega_{h, u}$ and $\sup _{\Omega}\left|u_{h}\right|=\sup _{\Omega_{h, u}}|u|$ for every $h>0$.

Moreover, $\left\{x \in \Omega: u_{h} \neq u\right\}=\Omega \backslash\left(\Omega_{h, u} \backslash E\right)$ and $|E|=0$.

Then

$$
\left|\left\{x \in \Omega: u_{h} \neq u\right\}\right|=\left|\Omega \backslash \Omega_{h, u}\right| .
$$

Since $\Omega_{h, u}$ is a measurable open bounded set, then for every $h>0$ there exists a closed set $F_{h} \subset \Omega_{h, u}$ such that $\left|\Omega_{h, u} \backslash F_{h}\right| \leq 1 / \Phi^{2}(h)$.

Then $D u \equiv D u_{h}$ a.e. in $F_{h}$; hence

$$
\left|\Omega \backslash F_{h}\right|=\left|\Omega \backslash \Omega_{h, u}\right|+\left|\Omega_{h, u} \backslash F_{h}\right| \leq\left|\Omega \backslash \Omega_{h, u}\right|+\frac{1}{\Phi^{2}(h)} .
$$

By (3.25),

$$
\lim _{h \rightarrow+\infty}\left|\Omega \backslash F_{h}\right|=0
$$

and Theorem 1.2(c) is proved.

When $\Phi \in \Delta_{2} \cap \nabla_{2}$, we will prove

$$
\lim _{h \rightarrow+\infty} \Phi(h)\left|\Omega \backslash F_{h}\right|=0 .
$$

In fact,

$$
\begin{aligned}
\Phi(h)\left|\Omega \backslash F_{h}\right| & =\Phi(h)\left|\Omega \backslash \Omega_{h, u}\right|+\Phi(h)\left|\Omega_{h, u} \backslash F_{h}\right| \\
& \leq \frac{2}{\Phi(h)}+\Phi(h)\left|\Omega \backslash \Omega_{h, u}\right| .
\end{aligned}
$$

Since $\Phi \in \Delta_{2}$, we can compute

$$
\begin{aligned}
\Phi(h) \mid & \left\{x \in \Omega: M^{\prime} u(x) \geq \frac{h}{c_{1}}\right\} \mid \\
& \leq c \Phi\left(\frac{h}{c_{1}}\right)\left|\left\{x \in \Omega: M^{\prime} u(x) \geq \frac{h}{c_{1}}\right\}\right| \\
& \leq c \Phi\left(\frac{h}{c_{1}}\right)\left|\left\{x \in \Omega: M \Phi(|D u(x)|) \geq \Phi\left(\frac{h}{c_{1}}\right)\right\}\right| \\
& \leq c \int_{\left\{x \in \Omega: M(\Phi(|D u|))(x) \geq \Phi\left(h / c_{1}\right)\right\}} M(\Phi(|D u|))(x) d x .
\end{aligned}
$$

By assumption, $\Phi \in \nabla_{2}$ and $\Phi(|D u|) \in L^{1}\left(\Omega, \mathbb{R}^{m}\right)$ and by Theorem 3.2, $M(\Phi(|D u|)) \in L^{1}(\Omega)$ holds, so we have

$$
\Phi(h)\left|\left\{x \in \Omega: M^{\prime} u(x) \geq \frac{h}{c_{1}}\right\}\right| \stackrel{h \rightarrow+\infty}{\longrightarrow} 0
$$


and we obtain (3.29). Finally for the monotonicity of $\Phi$ and the property of $u_{h}$, we have

$$
\left|D u_{h}\right| \leq h \Longrightarrow \Phi\left(\left|D u_{h}\right|\right) \leq \Phi(h) \Longrightarrow\left\|\Phi\left(\left|D u_{h}\right|\right)\right\|_{L^{\infty}\left(\Omega, \mathbb{R}^{m}\right)} \leq \Phi(h)
$$

which completes the proof.

\section{Young measures and Jensen inequality}

In this section, we give the proof of Theorem 1.2, based on arguments of the theory of Young measures. Hence we recall the most important properties; for the related proofs and particular results, we refer to $[3,7,13]$.

A Young measure is a family of probability measures $\nu=\left\{\nu_{x}\right\}_{x \in \Omega}$ associated with a sequence of functions $f_{j}: \Omega \subset \mathbb{R}^{n} \rightarrow \mathbb{R}^{m}$, such that $\operatorname{supp}\left(\nu_{x}\right) \subset \mathbb{R}^{m}$, depending measurably on $x \in \Omega$ in the sense that for any continuous $\phi: \mathbb{R}^{m} \rightarrow \mathbb{R}$, the function of $x$

$$
\bar{\phi}(x)=\int_{\mathbb{R}^{m}} \phi(\lambda) d v_{x}(\lambda)=\left\langle\phi, v_{x}\right\rangle
$$

is measurable.

If $\left\{u_{j}\right\}_{j \in \mathbb{N}}$ is a sequence of measurable functions $u_{j}: \Omega \rightarrow \mathbb{R}^{m}$ such that

$$
\sup _{j} \int_{\Omega} g\left(\left|u_{j}\right|\right) d x<+\infty
$$

where $g:[0,+\infty) \rightarrow[0,+\infty]$ is a continuous nondecreasing function and $\lim _{x \rightarrow+\infty} g(x)=+\infty$, then by Young existence theorem, there exist a subsequence, not relabeled, and a family of probability measures $\left\{v_{x}\right\}_{x \in \Omega}$ (the associated Young measure) depending measurably on $x$ with the property that whenever the sequence $\left\{\psi\left(x, u_{j}(x)\right)\right\}_{j \in \mathbb{N}}$ is weakly convergent in $L^{1}(\Omega)$ for any Carathéodory function $\psi(x, \lambda): \Omega \times \mathbb{R}^{m} \rightarrow \overline{\mathbb{R}}$, the weak limit is the function $\bar{\psi}(x)=$ $\int_{\mathbb{R}^{m}} \psi(x, \lambda) d \nu_{x}(\lambda)$.

Finally if we have that $u^{j}=\left(w^{j}, v^{j}\right): \Omega \rightarrow \mathbb{R}^{m} \times \mathbb{R}^{k}$ generates the Young measure $\left\{\mu_{x}\right\}_{x \in \Omega}, w^{j} \rightarrow w$ in measure and that the sequence $\left\{v^{j}\right\}_{j \in \mathbb{N}}$ generates the Young measure $\left\{v_{x}\right\}_{x \in \Omega}$, then for almost every $x \in \Omega$ we have $\mu_{x}=\delta_{w(x)} \otimes v_{x}$, which means that for any $f \in C_{0}\left(\mathbb{R}^{m} \times \mathbb{R}^{k}\right)$ and almost every $x \in \Omega$,

$$
\int_{\mathbb{R}^{m} \times \mathbb{R}^{k}} f(s, \lambda) d \mu_{x}(s, \lambda)=\int_{\mathbb{R}^{k}} f(w(x), \lambda) d \nu_{x}(\lambda) .
$$

Before giving the proof of Jensen inequality of Theorem 1.2 we need the following proposition. 
892 Semicontinuity by Young measures

Proposition 4.1. Let $\lambda \in \mathbb{R}^{m n}$ and let $f: \mathbb{R}^{m n} \rightarrow \mathbb{R}$ be a continuous function such that

$$
f(\lambda) \leq c(1+\Phi(|\lambda|))
$$

with $\Phi \in \Delta_{2} \cap \nabla_{2}$.

For $\left\{u^{j}\right\}_{j \in \mathbb{N}}$ in $W^{1, \phi, 1}\left(\Omega, \mathbb{R}^{m}\right)$ such that

$$
\sup _{j} \int_{\Omega} \Phi\left(\left|D u_{j}(x)\right|\right) d x \leq M
$$

let $u_{k}^{j} \in \operatorname{Lip}\left(\Omega, \mathbb{R}^{m}\right)$ be the Lipschitz function sequence of Theorem 1.3. Let $\left\{v_{x}\right\}_{x \in \Omega}$ and $\left\{\nu_{x}^{k}\right\}_{x \in \Omega}$ be the Young measures generated by the sequences $\left\{D u^{j}\right\}_{j \in \mathbb{N}}$ and $\left\{D u_{k}^{j}\right\}_{j \in \mathbb{N}}$, respectively. Then for all $\varepsilon>0$ there exists a subset $E \subset \Omega$ such that $|E|<\varepsilon$ and $\left\langle f, v_{x}^{k}\right\rangle \rightarrow\left\langle f, v_{x}\right\rangle$ in $L^{1}(\Omega \backslash E)$ as $k \rightarrow+\infty$.

Proof. Take $\varepsilon>0$; according to the Biting lemma (see [13]), we can find a set $E \subset \Omega$ such that $|E|<\varepsilon$ and $f\left(D u^{j}\right) \rightarrow \bar{f}$ in $L^{1}(\Omega \backslash E)$.

By the Young existence theorem, $\left\langle f, v_{x}\right\rangle$ is the weak limit of $f\left(D u^{j}\right)$; hence $\bar{f}=\left\langle f, v_{x}\right\rangle$ a.e. in $\Omega$. Then,

$$
\begin{aligned}
& \int_{\Omega \backslash E}\left|\left\langle f, v_{x}^{k}\right\rangle-\left\langle f, v_{x}\right\rangle\right| d x \\
&=\sup _{\|\psi\|_{L^{\infty} \leq 1}}\left|\int_{\Omega \backslash E} \psi(x)\left(\left\langle f, v_{x}^{k}\right\rangle-\left\langle f, v_{x}\right\rangle\right) d x\right| \\
&=\sup _{\|\psi\|_{L^{\infty} \leq 1}}\left|\lim _{j \rightarrow+\infty} \int_{\Omega \backslash E} \psi(x)\left(f\left(D u_{k}^{j}\right)-f\left(D u^{j}\right)\right) d x\right| \\
& \leq \sup _{\|\psi\|_{L^{\infty} \leq 1}} \lim _{j \rightarrow+\infty} \int_{\Omega \backslash E}|\psi(x)|\left|f\left(D u_{k}^{j}\right)-f\left(D u^{j}\right)\right| d x \\
&=\sup _{\|\psi\|_{L^{\infty}} \leq 1} \lim _{j \rightarrow+\infty} \int_{(\Omega \backslash E) \backslash F_{k}^{j}}|\psi(x)|\left|f\left(D u_{k}^{j}\right)-f\left(D u^{j}\right)\right| d x \\
& \quad+\sup _{\|\psi\|_{L^{\infty}} \leq 1} \lim _{j \rightarrow+\infty} \int_{(\Omega \backslash E) \cap F_{k}^{j}}|\psi(x)|\left|f\left(D u_{k}^{j}\right)-f\left(D u^{j}\right)\right| d x \\
& \leq \sup _{j} \int_{(\Omega \backslash E) \backslash F_{k}^{j}}\left|f\left(D u_{k}^{j}\right)-f\left(D u^{j}\right)\right| d x \\
& \leq \sup _{j} \int_{(\Omega \backslash E) \backslash F_{k}^{j}}\left|f\left(D u_{k}^{j}\right)\right| d x+\sup _{j} \int_{(\Omega \backslash E) \backslash F_{k}^{j}}\left|f\left(D u^{j}\right)\right| d x .
\end{aligned}
$$

Since

$$
\begin{aligned}
& \sup _{j} \int_{(\Omega \backslash E) \backslash F_{k}^{j}}\left|f\left(D u_{k}^{j}\right)\right| d x \\
& \quad \leq \sup _{j} \int_{(\Omega \backslash E) \backslash F_{k}^{j}} c\left(1+\Phi\left(\left|D u_{k}^{j}\right|\right)\right) d x
\end{aligned}
$$




$$
\begin{aligned}
& \leq \sup _{j} c\left|(\Omega \backslash E) \backslash F_{k}^{j}\right|+\sup _{j} \int_{(\Omega \backslash E) \backslash F_{k}^{j}} c \Phi\left(\left|D u_{k}^{j}\right|\right) d x \\
& \leq \sup _{j} c\left|(\Omega \backslash E) \backslash F_{k}^{j}\right|+\sup _{j} c \Phi(k)\left|(\Omega \backslash E) \backslash F_{k}^{j}\right|
\end{aligned}
$$

by Theorem 1.3 for all $j \in \mathbb{N}$,

$$
\left|(\Omega \backslash E) \backslash F_{k}^{j}\right| \stackrel{k \rightarrow+\infty}{\longrightarrow} 0, \quad \Phi(k)\left|(\Omega \backslash E) \backslash F_{k}^{j}\right| \stackrel{k \rightarrow+\infty}{\longrightarrow} 0 .
$$

For the second term of (4.6),

$$
\sup _{j} \int_{(\Omega \backslash E) \backslash F_{k}^{j}}\left|f\left(D u^{j}\right)\right| d x \leq \sup _{j} \int_{(\Omega \backslash E) \backslash F_{k}^{j}} c\left(1+\Phi\left(\left|D u^{j}\right|\right)\right) .
$$

Passing to the limit for $k$ in (4.7) and (4.9), we have $\left\langle f, v_{x}^{k}\right\rangle \rightarrow\left\langle f, v_{x}\right\rangle$ in $L^{1}(\Omega \backslash E)$ as $k \rightarrow+\infty$ and the theorem is proved.

Finally we get the Jensen's inequality.

Proof of Theorem 1.2. We can assume that $\Omega$ is a ball of $\mathbb{R}^{n}$.

For the proof of (1.8), we define the Carathéodory function $\Psi^{(h, s)}: \Omega \times \mathbb{R}^{m n} \rightarrow$ $\mathbb{R}$, with $\Psi^{(h, s)}(x, \lambda)=\lambda_{h, s}$, where $\lambda_{h, s}$ is the element in position $(h, s)$ of the ma$\operatorname{trix} \lambda \in \mathbb{R}^{m n}$. Then for what recalled about Young measures, $\Psi^{(h, s)}\left(x, D u^{j}(x)\right) \rightarrow$ $\bar{\Psi}^{(h, s)}(x)=\int_{\mathbb{R}^{m n}} \Psi^{(h, s)}(x, \lambda) d v_{x}(\lambda)$ holds, as $j \rightarrow+\infty$, in $L^{1}(\Omega)$, and by hypothesis, $\Psi^{(h, s)}\left(x, D u^{j}(x)\right) \rightarrow(D u(x))_{h, s}$ as $j \rightarrow+\infty$. So by uniqueness of weak limit we have

$$
(D u(x))_{h, s}=\int_{\mathbb{R}^{m n}} \lambda_{h, s} d \nu_{x}(\lambda) \quad \text { for almost every } x \in \Omega,
$$

for all $h=1,2, \ldots, m$ and $s=1,2, \ldots, n$, then

$$
D u(x)=\int_{\mathbb{R}^{m n}} \lambda d \nu_{x}(\lambda) \quad \text { for almost every } x \in \Omega .
$$

Now we divide the proof into 4 steps.

Step 1. Suppose that $f=f(\lambda)$ is continuous and $u^{j} \in W^{1, \infty}\left(\Omega, \mathbb{R}^{m}\right)$ and take $x \in \Omega$ and $r>0$ such that $Q(x, r) \subset \Omega$. Let $\sigma$ be a constant with $0<\sigma<r$ and $\phi_{\sigma} \in C_{0}^{\infty}(Q(x, r))$, where $\phi_{\sigma} \equiv 1$ on $Q(x, r-\sigma)$. Applying standard arguments, the function $w_{\sigma}^{j}=\phi_{\sigma} \cdot\left(u^{j}-u\right)$ can be substituted in the definition of quasiconvexity; hence we have

$$
f(A) \leq \frac{1}{|Q(x, r)|} \int_{Q(x, r)} f\left(A+D \phi_{\sigma} \cdot\left(u^{j}-u\right)+\phi_{\sigma} \cdot\left(D u^{j}-D u\right)\right) d y
$$

for all $A \in \mathbb{R}^{m n}$. 
Let

$$
I(x, r, \sigma, j)=\frac{1}{|Q(x, r)|} \int_{Q(x, r)} f\left(A+D \phi_{\sigma} \cdot\left(u^{j}-u\right)+\phi_{\sigma} \cdot\left(D u^{j}-D u\right)\right) d y .
$$

Since $\left\{D w_{\sigma}^{j}\right\}$ is uniformly bounded on $\Omega$, the sequence $\left\{f\left(A+D w_{\sigma}^{j}\right)\right\}_{j \in \mathbb{N}}$ is relatively compact in $L^{1}(\Omega)$. We define

$$
v^{j}=\left(u^{j}-u, D u^{j}\right): \Omega \longrightarrow \mathbb{R}^{m} \times \mathbb{R}^{m n},
$$

which generates the Young measure $\left\{\mu_{y}\right\}_{y \in \Omega}$; furthermore, $u^{j}-u \rightarrow 0$ in $L^{1}(\Omega)$ as $j \rightarrow+\infty$ and $D u^{j}$ generates the Young measure $\left\{\nu_{y}\right\}_{y \in \Omega}$; then for almost every $y \in \Omega, \mu_{y}=\delta_{0} \otimes \nu_{y}$ holds. If we consider $\bar{g}(s, \lambda)=f\left(A+D \phi_{\sigma} \cdot s+\phi_{\sigma} \cdot(\lambda-D u)\right)$, where $s=u^{j}-u$ and $\lambda=D u^{j}$, we have $|\bar{g}(s, \lambda)| \leq K$, where $K$ is a constant depending only on $A$, hence the following holds:

$$
\int_{\mathbb{R}^{m} \times \mathbb{R}^{m n}} \bar{g}(s, \lambda) d \mu_{y}(s, \lambda)=\int_{\mathbb{R}^{m n}} \bar{g}(0, \lambda) d \nu_{y}(\lambda) .
$$

Furthermore, the Young existence theorem gives

$$
\bar{g}(s, \lambda) \rightarrow \int_{\mathbb{R}^{m} \times \mathbb{R}^{m n}} \bar{g}(s, \lambda) d \mu_{y}(s, \lambda) .
$$

Then, by the definition of $\bar{g}$, the following holds:

$$
f\left(A+D \phi_{\sigma} \cdot s+\phi_{\sigma} \cdot(\lambda-D u)\right)-\int_{\mathbb{R}^{m n}} f\left(A+\varphi_{\sigma}(\lambda-D u)\right) d v_{y}(\lambda) .
$$

Hence by (4.17), we have

$$
\begin{aligned}
I(x, r, \sigma, j) & \stackrel{j \rightarrow+\infty}{\longrightarrow} \frac{1}{|Q(x, r)|} \int_{Q(x, r)} \int_{\mathbb{R}^{m n}} f\left(A+\varphi_{\sigma}(\lambda-D u)\right) d \nu_{y}(\lambda) d y \\
& =I(x, r, \sigma),
\end{aligned}
$$

where $v_{y}$ is supported on a bounded set. Applying the Lebesgue convergence theorem and taking the limit on $\sigma$, we have

$$
I(x, r, \sigma) \longrightarrow \frac{1}{|Q(x, r)|} \int_{Q(x, r)} \int_{\mathbb{R}^{m n}} f(A+\lambda-D u) d \nu_{y}(\lambda) d y .
$$

By the theorem about the Lebesgue's points for every $A \in \mathbb{R}^{m n}$, a set $\Omega(A) \subset \Omega$ exists such that $|\Omega \backslash \Omega(A)|=0$ and for all $x \in \Omega(A)$,

$$
f(A) \leq \int_{\mathbb{R}^{m n}} f(A+\lambda-D u) d \nu_{y}(\lambda) .
$$

Furthermore, we can suppose that $\sup _{x \in \Omega(A)}|D u(x)|<\infty$. Let $\left\{A^{j}\right\}$ be a dense and countable subset of $\mathbb{R}^{m n}$. 
Consider now the set $\Omega_{1}=\bigcap_{j} \Omega\left(A^{j}\right)$ of full measure in $\Omega$

$$
\left|\Omega \backslash \Omega_{1}\right|=\left|\bigcup_{j} \Omega \backslash \Omega\left(A^{j}\right)\right| \leq \sum_{j}\left|\Omega \backslash \Omega\left(A^{j}\right)\right|=0 .
$$

Hence for every $x \in \Omega_{1}$, (4.20) holds with $A=A^{j}$. Let $x \in \Omega_{1}$, then by density, there exists a subsequence $A^{j_{k}}$ which converges to $D u(x) \in \mathbb{R}^{m n}$ as $k \rightarrow+\infty$. Finally, it is sufficient to note that $f\left(A^{j_{k}}\right) \rightarrow f(D u(x))$ by continuity of $f$, and

$$
\int_{\mathbb{R}^{m n}} f\left(A^{j_{k}}-D u(x)+\lambda\right) d v_{x}(\lambda) \longrightarrow \int_{\mathbb{R}^{m n}} f(\lambda) d v_{x}(\lambda)
$$

Furthermore, by the equality $D u(x)=\int_{\mathbb{R}^{m n}} \lambda d \nu_{x}(\lambda)=\left\langle\lambda, v_{x}\right\rangle$ and by (4.20) we have

$$
\begin{aligned}
f(D u(x)) & =\lim _{k} f\left(A^{j_{k}}\right) \leq \int_{\mathbb{R}^{m n}} f\left(A^{j_{k}}-D u(x)+\lambda\right) d \nu_{x}(\lambda) \\
& =\int_{\mathbb{R}^{m n}} f(\lambda) d \nu_{x}(\lambda),
\end{aligned}
$$

and we can conclude that

$$
f\left(\int_{\mathbb{R}^{m n}} \lambda d v_{x}(\lambda)\right) \leq \int_{\mathbb{R}^{m n}} f(\lambda) d v_{x}(\lambda)
$$

Step 2. Suppose that $f=f(\lambda)$ is continuous and $u^{j} \in W^{1, \Phi, 1}\left(\Omega, \mathbb{R}^{m}\right)$.

By the approximation theorem (Theorem 1.3) applied at $u \in W^{1, \Phi, 1}\left(\Omega, \mathbb{R}^{m}\right)$, we have for every $k>0$ a function $u_{k} \in \operatorname{Lip}\left(\Omega, \mathbb{R}^{m}\right)$ such that $\left\|\Phi\left(\left|D u_{k}\right|\right)\right\|_{L^{\infty}\left(\Omega, \mathbb{R}^{m}\right)} \leq$ $\Phi(k), D u \equiv D u_{k}$ a.e. on $F_{k}$ with $F_{k} \subset \Omega$ closed set such that $\Phi(k)\left|\Omega \backslash F_{k}\right| \rightarrow 0$ as $k \rightarrow+\infty$.

Applying Theorem 1.3 to $u^{j}$, we obtain the function $u_{k}^{j}$. Note that $u_{k}^{j} \in \operatorname{Lip}(\Omega$, $\mathbb{R}^{m}$ ) verifies the assumptions in Step 1, then the following holds:

$$
f\left(\left\langle\lambda, v_{x}^{k}\right\rangle\right) \leq \int_{\mathbb{R}^{m n}} f(\lambda) d v_{x}^{k}(\lambda)
$$

where $\left\{v_{x}^{k}\right\}_{x \in \Omega}$ is the Young measure generated by $\left\{D u_{k}^{j}\right\}_{j}$ and $\lambda$ is the identity function $g(\lambda)=\lambda$. Hence

$$
f\left(\left\langle\lambda, v_{x}^{k}\right\rangle\right)=f\left(\int_{\mathbb{R}^{m n}} \lambda d v_{x}^{k}(\lambda)\right)
$$

By Proposition 4.1 for $g(\lambda)=\lambda$, we have

$$
\left\langle\lambda, v_{x}^{k}\right\rangle \longrightarrow\left\langle\lambda, v_{x}\right\rangle
$$


896 Semicontinuity by Young measures

as $k \rightarrow+\infty$. In the left-hand side of (4.25), by continuity of $f$, we have

$$
f\left(\left\langle\lambda, v_{x}^{k}\right\rangle\right) \stackrel{k \rightarrow+\infty}{\longrightarrow} f\left(\left\langle\lambda, v_{x}\right\rangle\right)
$$

a.e. in $\mathbb{R}^{m n}$. Applying Proposition 4.1, we obtain

$$
\left\langle f, v_{x}^{k}\right\rangle \stackrel{k \rightarrow+\infty}{\longrightarrow}\left\langle f, v_{x}\right\rangle
$$

on $\mathbb{R}^{m n}$. Finally, passing to the limit as $k \rightarrow+\infty$ in $(4.25)$, we have proved that

$$
f\left(\left\langle\lambda, v_{x}\right\rangle\right) \leq\left\langle f, v_{x}\right\rangle
$$

then for (4.26),

$$
f\left(\int_{\mathbb{R}^{m n}} \lambda v_{x}(\lambda)\right) \leq \int_{\mathbb{R}^{m n}} f(\lambda) v_{x}(\lambda)
$$

Step 3. Let $f=f(x, \lambda)$ be continuous and $u^{j} \in W^{1, \Phi, 1}\left(\Omega, \mathbb{R}^{m}\right)$, and let $E(x) \leq$ $L<+\infty$. If $\left\{b_{j}\right\}_{j \in \mathbb{N}}$ is a dense set in $\Omega$, for every $j \in \mathbb{N}$, define $f^{j}=f\left(b^{j}, \lambda\right)$; so by Step 2 there exists $\Omega_{1}^{j} \subset \Omega$ with $\left|\Omega \backslash \Omega_{1}^{j}\right|=0$ such that for all $x \in \Omega_{1}^{j}$, we have

$$
f\left(b^{j}, \int_{\mathbb{R}^{m n}} \lambda d v_{x}(\lambda)\right) \leq \int_{\mathbb{R}^{m n}} f\left(b^{j}, \lambda\right) d v_{x}(\lambda)
$$

Define

$$
\Omega_{1}=\bigcap_{j=1}^{\infty} \Omega_{1}^{j},
$$

then $\left|\Omega \backslash \Omega_{1}\right|=0$. For every fixed $x \in \Omega_{1}$, by density of $\left\{b_{j}\right\}_{j \in \mathbb{N}}$, there exists a subsequence $b^{j_{k}}$ which converges to $x$ as $k \rightarrow+\infty$; then by continuity of $f$ and by dominated convergence theorem the following holds:

$$
\begin{gathered}
f\left(b^{j_{k}}, \int_{\mathbb{R}^{m n}} \lambda d v_{x}(\lambda)\right) \stackrel{k \rightarrow+\infty}{\longrightarrow} f\left(x, \int_{\mathbb{R}^{m n}} \lambda d v_{x}(\lambda)\right), \\
\left|f\left(b^{j_{k}}, \lambda\right)\right| \leq\left|E\left(b^{j_{k}}\right)\right||1+\Phi(|\lambda|)| \leq L(1+\Phi(|\lambda|)) .
\end{gathered}
$$

Hence

$$
\int_{\mathbb{R}^{m n}} f\left(b^{j_{k}}, \lambda\right) d \nu_{x}(\lambda) \stackrel{k \rightarrow+\infty}{\longrightarrow} \int_{\mathbb{R}^{m n}} f(x, \lambda) d \nu_{x}(\lambda) .
$$


Passing to the limit on $k \rightarrow+\infty$ in (4.32), we have

$$
f\left(x, \int_{\mathbb{R}^{m n}} \lambda d \nu_{x}(\lambda)\right) \leq \int_{\mathbb{R}^{m n}} f(x, \lambda) d \nu_{x}(\lambda)
$$

Step 4. Let now $f(x, \lambda)$ be a Carathéodory function such that, for almost every $x \in \Omega,|f(x, \lambda)| \leq E(x)(1+\Phi(|\lambda|))$ is verified. Applying the Scorza-Dragoni theorem to $f(x, \lambda)$ and the Lusin theorem to $E(x)$, there exists for every $\varepsilon>0$ a compact set $\Omega_{\varepsilon}$ with $\left|\Omega \backslash \Omega_{\varepsilon}\right|<\varepsilon$ such that $f(x, \lambda)$ is continuous in $\Omega_{\varepsilon} \times \mathbb{R}^{m n}$ and $E(x)$ is bounded in $\Omega_{\varepsilon}$. For Step 3, we obtain the assertion in $\Omega_{\varepsilon}$ and then in $\Omega$. Hence (1.7) is proved.

\section{Acknowledgment}

The author thanks Prof. E. Mascolo for her useful advice.

\section{References}

[1] E. Acerbi and N. Fusco, An approximation lemma for $W^{1, p}$ functions, Material Instabilities in Continuum Mechanics (Edinburgh, 1985-1986), Oxford Sci. Publ., Oxford University Press, New York, 1988, pp. 1-5.

[2] R. A. Adams, Sobolev Spaces, Pure and Applied Mathematics, vol. 65, Academic Press, New York, 1975.

[3] J. M. Ball, A version of the fundamental theorem for Young measures, PDEs and Continuum Models of Phase Transitions (Nice, 1988) (M. Rascle, D. Serre, and M. Slemrod, eds.), Lecture Notes in Phys., vol. 344, Springer-Verlag, Berlin, 1989, pp. 207-215.

[4] B. Bianconi, M. Focardi, and E. Mascolo, Existence of minimizers for a class of quasi-convex functionals with non-standard growth, Ann. Mat. Pura Appl. (4) 180 (2002), no. 4, 493-510.

[5] N. Fusco, Quasi-convessità e semicontinuità per integrali multipli di ordine superiore [Quasiconvexity and semicontinuity for higher-order multiple integrals], Ricerche Mat. 29 (1980), no. 2, 307-323 (Italian).

[6] J.-P. Gossez, Some approximation properties in Orlicz-Sobolev spaces, Studia Math. 74 (1982), no. 1, 17-24.

[7] A. Kałamajska, On lower semicontinuity of multiple integrals, Colloq. Math. 74 (1997), no. 1, 71-78.

[8] V. Kokilashvili and M. Krbec, Weighted Inequalities in Lorentz and Orlicz Spaces, World Scientific Publishing, New Jersey, 1991.

[9] M. A. Krasnosel'skiı̌ and Ja. B. Rutickiı̌, Convex Functions and Orlicz Spaces, P. Noordhoff, Groningen, 1961.

[10] A. Kufner, O. John, and S. Fučík, Function Spaces Mechanics: Analysis, Monographs and Textbooks on Mechanics of Solids and Fluids, Academia, Prague, 1977.

[11] P. Marcellini, Approximation of quasi-convex functions, and lower semicontinuity of multiple integrals, Manuscripta Math. 51 (1985), no. 1-3, 1-28.

[12] E. Mascolo and G. Papi, Harnack inequality for minimizers of integral functionals with general growth conditions, NoDEA Nonlinear Differential Equations Appl. 3 (1996), no. 2, 231-244. 
[13] P. Pedregal, Parametrized Measures and Variational Principles, Progress in Nonlinear Differential Equations and Their Applications, vol. 30, Birkhäuser Verlag, Basel, 1997.

[14] M. M. Rao and Z. D. Ren, Theory of Orlicz Spaces, Monographs and Textbooks in Pure and Applied Mathematics, vol. 146, Marcel Dekker, New York, 1991.

[15] E. M. Stein, Singular Integrals and Differentiability Properties of Functions, Princeton Mathematical Series, no. 30, Princeton University Press, New Jersey, 1970.

Barbara Bianconi: Dipartimento di Scienze Matematiche, Universitá Politecnica delle Marche, Via Brecce Bianche, 60122 Ancona, Italy

E-mail address: bianconi@dipmat.unian.it 


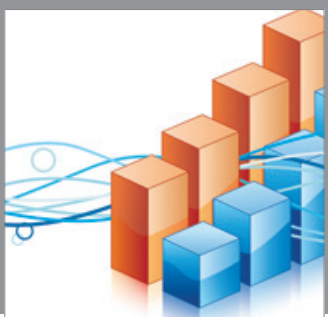

Advances in

Operations Research

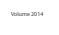

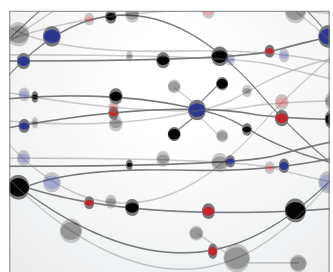

\section{The Scientific} World Journal
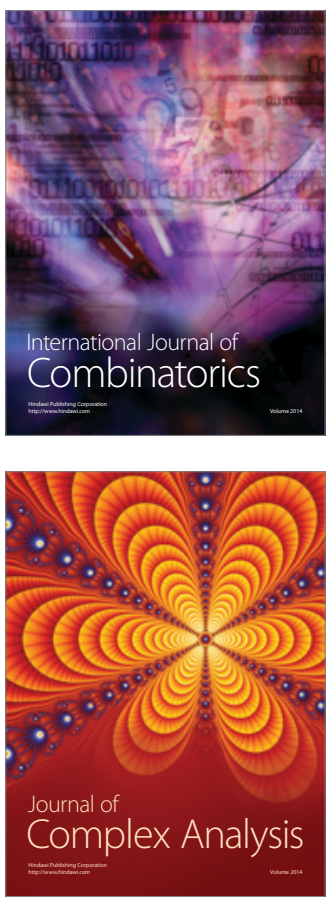

International Journal of

Mathematics and

Mathematical

Sciences
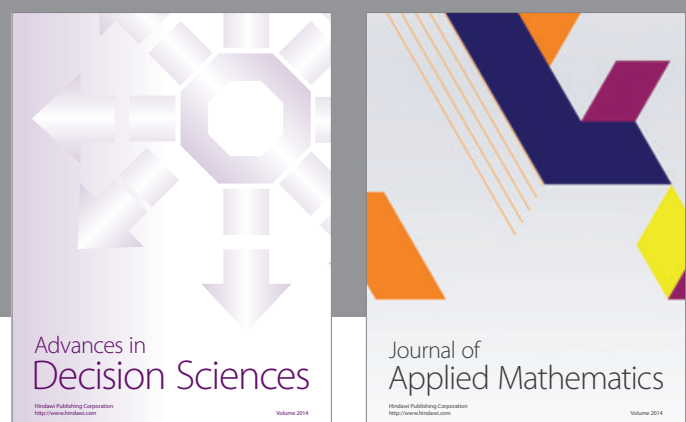

Journal of

Applied Mathematics
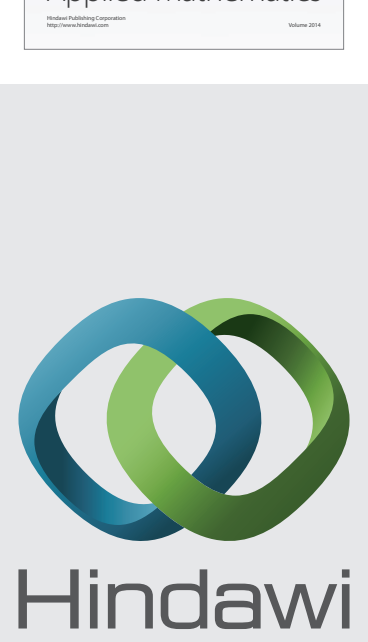

Submit your manuscripts at http://www.hindawi.com
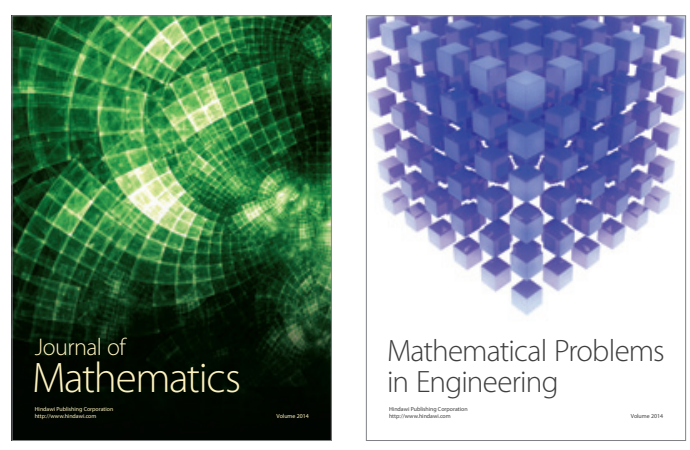

Mathematical Problems in Engineering
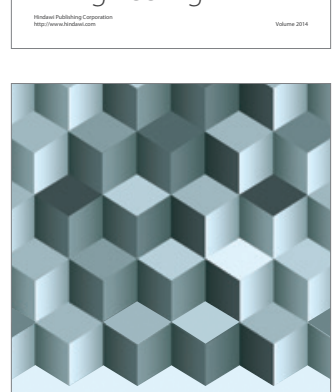

Journal of

Function Spaces
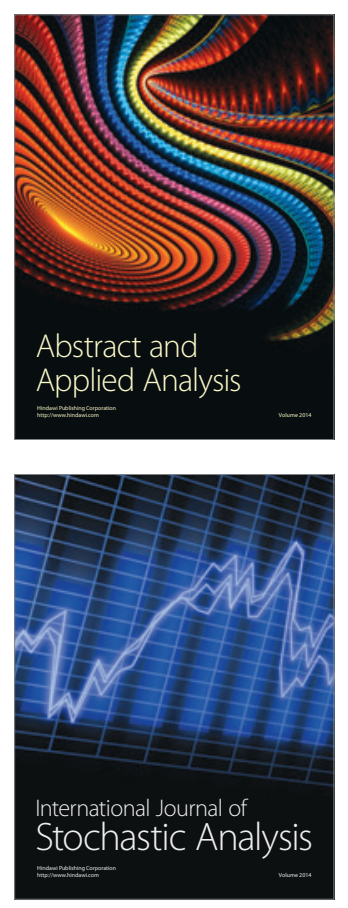

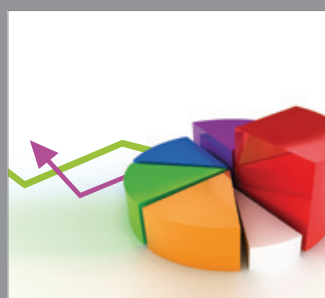

ournal of

Probability and Statistics

Promensencen
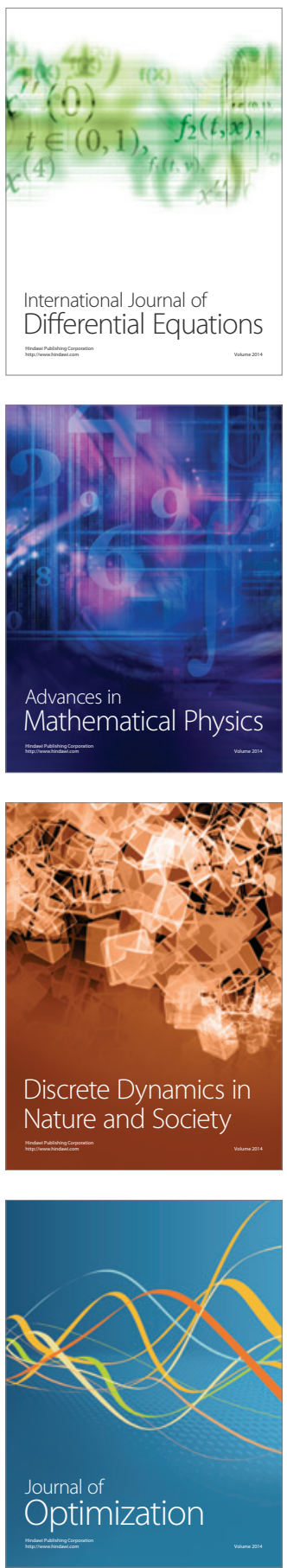